\title{
Towards Efficient Parallel Image Processing on Cluster Grids Using GIMP ${ }^{\star}$
}

\author{
Paweł Czarnul, Andrzej Ciereszko, and Marcin Frączak \\ Faculty of Electronics, Telecommunications and Informatics \\ Gdansk University of Technology, Poland \\ pczarnul@eti.pg.gda.pl, \{cierech,marcin.f\}@wp.pl \\ http://fox.eti.pg.gda.pl/ pczarnul
}

\begin{abstract}
As it is not realistic to expect that all users, especially specialists in the graphic business, use complex low-level parallel programs to speed up image processing, we have developed a plugin for the highly acclaimed GIMP which enables to invoke a series of filter operations in a pipeline in parallel on a set of images loaded by the plugin. We present the software developments, test scenarios and experimental results on cluster grid systems possibly featuring single-processors and SMP nodes and being used by other users at the same time. Behind the GUI, the plugin invokes a smart DAMPVM cluster grid shell which spawns processes on the best nodes in the cluster, taking into account their loads including other user processes. This enables to select the fastest nodes for the stages in the pipeline. We show by experiment that the approach prevents scenarios in which other user processes or even slightly more loaded processors become the bottlenecks of the whole pipeline. The parallel mapping is completely transparent to the end user who interacts only with the GUI. We present the results achieved with the GIMP plugin using the smart cluster grid shell as well as a simple round robin scheduling and prove the former solution to be superior.
\end{abstract}

\section{Introduction}

While both the cluster and grid architectures ([1]) and real grid software based on Globus ([2]) as well as image processing tools become mature and available for a wider range of systems and network topologies, it is still a difficult task to merge the two worlds in open NOWs. We investigate available solutions and make an attempt to process in parallel both loosely coupled and pipelined images on NOW systems. In the context of parallel image processing there is a need for an easy-to-use graphical user interface of a familiar application like Adobe Photoshop or the GIMP and an efficient but not too complex a tool for selection of best resources. Ideally, as we managed to achieve, the second stage is completely hidden from the user provided the prior configuration of the parallel environment. Following the Sun Grid Engine terminology ([3]) we define the cluster grid as a set of distributed resources providing a single point of entry (running GIMP in our approach) to users in an institution.

\footnotetext{
* Work partially sponsored by the Polish National Grant KBN No. 4 T11C 00525
} 


\section{Related Work}

System or application level solutions which could assist in running either sequential or parallel image processing codes in parallel, range from system-level Mosix ([4]) which features process migration for load balancing for Linux boxes, cluster management systems like LoadLeveler, PBS, LSF ([5]) Condor ([6]) for exploiting idle cycles in shared networks up to Sun Grid Engine ([3]) which enables both queueing submitted HPC tasks and launching interactive processes on least loaded nodes allowing grid computing. There are libraries and environments like MPI, PVM ([7]) available for programmers. MatlabMPI ([8]) implements the MPI-like programming API for Matlab scripts to be run on parallel systems using the file system for communication. Although the latter three can assist in writing parallel image processing, none of them is by design coupled to specific parallel applications, including graphic design.

In regard to support for multithreaded image processing, in applications like Adobe Photoshop on SMP machines some filters like Gaussian Blur, Radial Blur, Image Rotate, Unsharp Mask can potentially benefit from many processors ([9]). However, some filters which can be accomplished fast can run even slower on SMP boxes than on a single processor due to the large synchronization overhead compared to the processing time. It has been confirmed that the multithreaded approach can speed up processing on the latest Intel HyperThreading processors ([10]) when running filters in Photoshop 7.0. [11] presents threaded GIMP plugins implementing Gaussian Blur.

[12] assumes, similarly to our approach, that it is unrealistic to expect the knowledge on parallelization issues from graphic specialists and provides the programmer with the architecture in which data parallel image processing applications can be coded sequentially, automatically parallelized and run on a homogeneous cluster of machines.

[13] presents a skeleton based approach for data parallel image processing in which only algorithmic skeletons coded in C/MPI need to be chosen for particular given lowlevel image operators to produce a parallel version of the code.

Finally the Parallel Image Processing Toolkit (PIPT, [14]) provides an extendable framework assisting image processing offering low-level API which can be incorporated into parallel MPI programs. This allows operations on chunks of images in parallel. Similarly, [15] describes a library of functions SPMDlib meant to help the development of image processing algorithms and a set of directives SPMDdir which are mapped by a parser to the library which provides an easy-to-use and high-level API.

\section{Our Approach for Cluster Parallel Image Processing}

In order to show the benefits of cluster computing in an open multi-user NOW, we have implemented an extension to the DAMPVM environment ([16], [17]) which provides the end user with a smart cluster shell which starts a shell command or a set of commands on best nodes in the network. Compared to Sun Grid Engine, it supports any platform PVM can run on, not only Solaris Operating Environments (on SPARC processors) and Linux x86 for Sun Grid Engine 5.3. Moreover, the DAMPVM sources can be modified to use a cluster even more efficiently. In view of this, the presented solution can easily use the monitoring/filtering infrastructure ([18]) and the divide-and-conquer features ([17]) of 
DAMPVM. Thus it has been naturally extended to enable the GIMP plugin to launch processes on least loaded processors in a parallel environment incorporating run-time changes into decision making. The plugin can make use of the GIMP support for reading and writing various graphic formats like TIFF, PNG, JPEG etc. and leave the computing part for the efficient $\mathrm{C}++$ code using PVM or other means of communication like MPI.

To prove the usefulness of this approach we have tested the following scenarios:

1. Starting command-line multi process conversion (using ImageMagick's convert utility) of large images in a network with varying loads. The cluster shell selects the least loaded nodes for convert to be run and thus optimizes the wall time of the simulation.

2. A GIMP plugin which invokes parallel pipelined processing using PVM applying a sequence of filters on images read by GIMP, all implemented by us within this work 1 . In corresponding external conditions, we prove that the allocation of pipeline processes to processors using the DAMPVM cluster shell can lead to a noticeable reduction of the execution time compared to round-robin/random allocation. This is visible even in lightly loaded networks where the DAMPVM shell chooses the least loaded nodes. In the pipeline, even small, difficult to notice processor loads appear to be a bottleneck for the pipeline which justifies our approach.

\subsection{Speed Measurement and Task Queueing}

In a heterogeneous network different but comparable processors like AMD Athlon XPs, MPs, Intel Pentium 4 (HT) can process different codes at different speeds. Secondly, the load of other users must be measured and filtered in order to hide high-frequency peaks corresponding to short-lived but CPU-bound actions like starting Mozilla etc. The latter was implemented in the DAMPVM runtime before ([18]). Within the scope of this work it was extended with precise speed measurement for specific code operations to reflect the real CPU-bound long application which follows the measurement. This can be the same processing command used on smaller images.

We applied the algorithm shown in Figure 1 to the DAMPVM cluster shell. When there are idle processors in the mixed single-processor, SMP system (as indicated by DAMPVM schedulers) the relevant information is stored in an array and the idle processors are assigned tasks successively with no delays. After the procedure has been completed, a 10-second delay is introduced to allow for the increased load being detected by the DAMPVM runtime. When all processors are still busy, every 2-second time slot a load check is performed. The values result from arbitrary delays to monitor load coded in the DAMPVM runtime. This scheme allows to use idle processors at once and queue pending supposedly processor and disk-bound image processing tasks in order not to overload the processor with too many processes resulting in process switching overhead. The load is monitored on every host by DAMPVM schedulers ([18]) and then collected asynchronously by a cluster manager using ring fashion communication. The load information includes: machine speed, the number of CPUs per node, idle percentages of the processors, CPU load by other users and the system, the number of processes, file storage available on the node, link start-up times and bandwidth.

\footnotetext{
${ }^{1}$ Download software from http://fox.eti.pg.gda.pl/ p pczarnul
} 


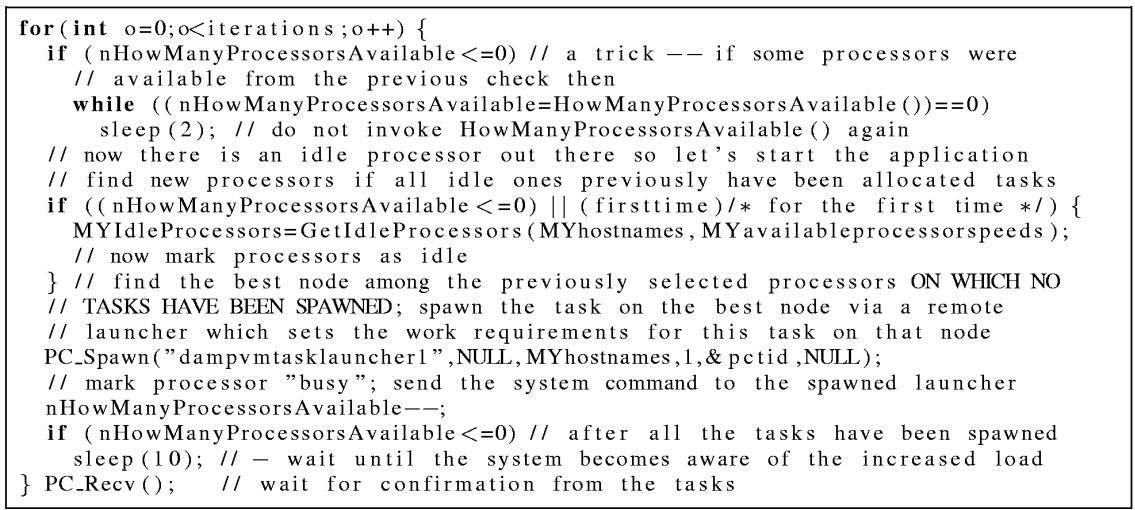

Fig. 1. Task Queueing in DAMPVM Remote Shell

\subsection{A GIMP Plugin for Pipelined Operations on Clusters}

The main idea of the plug-in is to apply a set of up to ten filters to a large number of images. The images should be placed on one machine with the GIMP, connected via network to a cluster of computers running PVM, a cluster grid. The architecture of the plug-in consists of three layers (Figure2):

- a Script-Fu wrapper,

- a supervisory GIMP-deployed plug-in,

- slave node modules in the proposed parallel pipelined architecture.

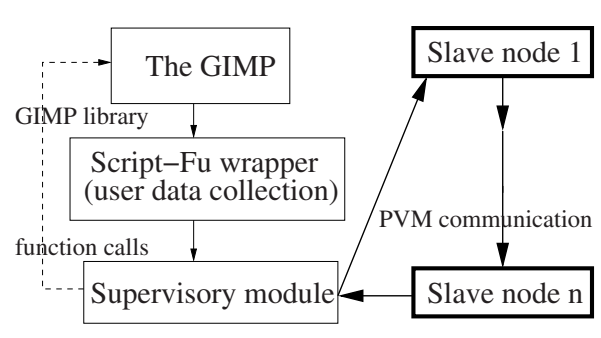

Fig. 2. GIMP Plugin's Architecture

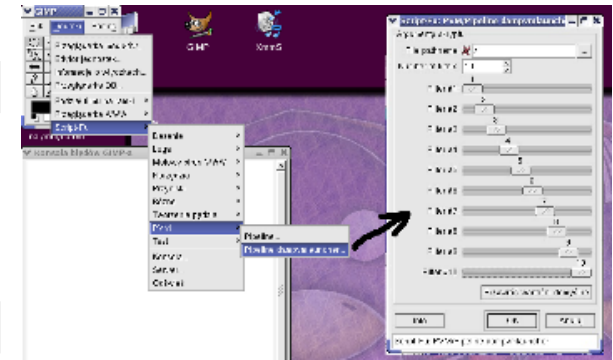

Fig. 3. GIMP's Pipelined Plugin Interface

The GIMP provides developers with an easy to use scripting language called Script$\mathrm{Fu}$, that is based on a scheme programming language. From the Script-Fu level you can run any of the GIMP library function including GIMP plug-ins properly registered in the PDB (GIMP's Procedural DataBase). Script-Fu also aids developers in creating of plug-in interfaces. The wrapper's function is to gather data from the user and relay them to the supervisory GIMP-deployed plug-in. 
Both, the supervisory module and the slave module, are programs written in $\mathrm{C}$ using PVM for communication. The supervisory module calls the GIMP's library functions to open each image for processing, acquires raw picture data from the GIMP's structures, and passes it to the first of the pipeline nodes. This allows to combine the easy-to-use interface with the underlying parallel architecture. The images are then processed in the created slave node pipeline. The pipeline can be created in two ways: either by using PVM (Pipeline plug-in) in accordance with a static list of hosts created by the user, or dynamically, with assistance of PVM based DAMPVM remote shell (Pipeline dampvmlauncher plug-in) thus taking advantage of the speed and load information of individual nodes in creating the slave node pipeline.

The slave node module implements a series of image filters $(3 \times 3,5 \times 5$ matrix area filters and simple non-context filters). Each slave applies a filter to the image and passes it on through the pipeline.

The interfaces for both plug-ins are identical, the difference lies in the code that is implemented in the supervisory module. The user invokes the plugin from the GIMP's context menu and specifies a path name for the files intended for filtering and the number of filters that will be applied to all the images. Figure 3 shows the plugin window superimposed on the context menu invoking it. Slide bars let the user specify the types of filters at the stages of the pipeline.

\section{Experimental Results}

We have performed lab experiments to prove that image processing on cluster grids can be successfully assisted by the load-aware remote DAMPVM shell. We used a cluster of 16 Intel Celeron $2 \mathrm{GHz}, 512 \mathrm{MB}$ machines interconnected with 100Mbps Ethernet.

\subsection{Parallel Image Conversion with a Cluster Shell}

In this case, we tested the ability of the DAMPVM runtime and the remote shell to detect least loaded nodes, spawn tasks remotely, queue pending tasks as described above and submit them when processors become idle. These features were tested in the scenario with one node loaded with other CPU-bound processes for which the results are shown in Figure 4. We ran ImageMagick's convert utility to convert a $4000 \times 400048 \mathrm{MB}$ TIFF image to Postscript. On 1 processor, we ran the command without the re-

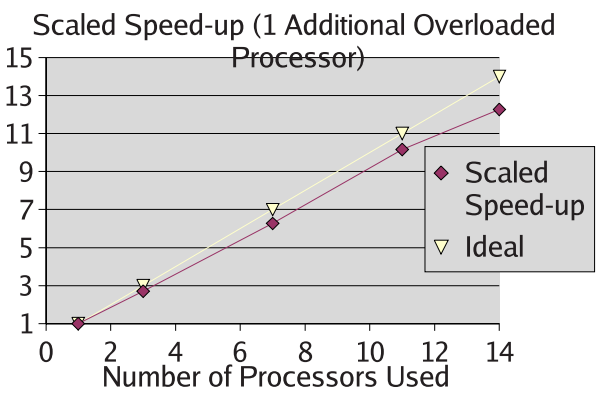

Fig. 4. Scaled Speed-up for Remote Shell convert Runs mote shell. On $P>1$ processors in Figure 4 there were $P+1$ processors available, one of which was overloaded. The remote shell omitted the overloaded node. The scaled speed-up was computed as the single-processor execution time multiplied by $P$ divided 
by the time achieved on $P$ processors with the load on the $P+1$-th processor. The latter processor was chosen randomly. Less than ideal values but showing small overhead result from additional load monitoring, spawn and the queueing procedure.

\subsection{Pipelined Image Filtering as a GIMP Plugin Using a Cluster}

In this case we compared launching pipelined computations using a static allocation of pipeline stages to processors and the dynamic allocation using the remote shell.

In the case that work is distributed in an unloaded and homogeneous network the efficiency of both plugins should be similar. However, in a normal network environment such conditions are hard to achieve and thus it is expected that total work times will be considerably shorter for the DAMPVM module. It is not only in academic examples in which some node(s) is overloaded but also in a seemingly idle network. In the latter case, some system activities or a user browsing the Internet contribute to the processor usage and such a node becomes effectively the pipeline bottleneck if selected as a stage of the pipeline. The remote shell enabled the plugin to avoid placing pipeline nodes on machines that are already loaded with work (e.g. the computer running the GIMP).

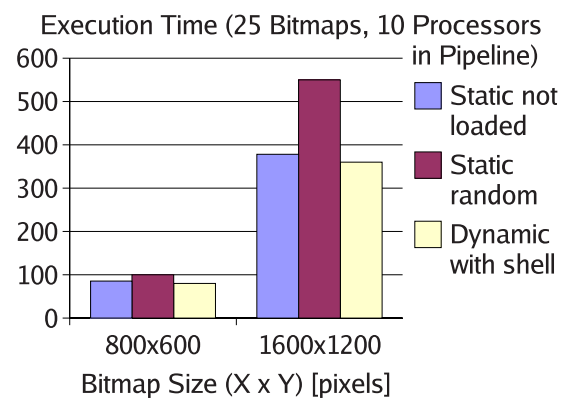

Fig. 5. Execution Time for Various Pipeline Allocation Methods by Image Size

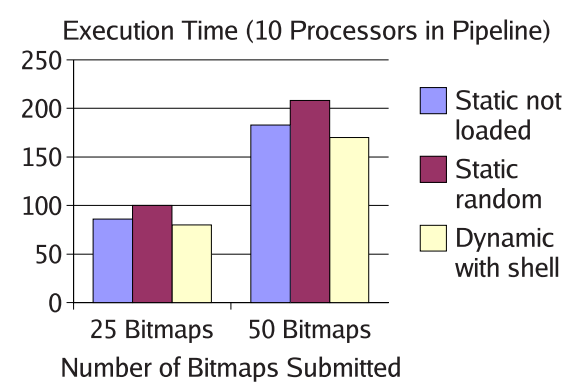

Fig. 6. Execution Time for Various Pipeline Allocation Methods by Number of Images

The variables in the pipelined simulations are as follows:

- the number of stages/processors in the pipeline $(P)$,

- the number of images to process $(N)$,

- the size of the images: may be of similar or different sizes,

- the type of filters at the stages: may be uniform or different in regard to the processing time.

Figure 5 presents results for $3 \times 3$ matrix filters (taking same time to complete) on $P=10$ processors for $N=25800 \times 600$ and $1600 \times 1200$ bitmaps while Figure 6 shows results for for $5 \times 5$ matrix filters (taking same time to complete) on $P=10$ processors for $N=25$ and $N=50800 \times 600$ bitmaps. In all cases there were 14 idle processors available. On one of them there was the GIMP running, acting as a master host, on another one a user performed simple editing in Emacs. In the "Static not loaded" 
case, the allocation was done statically by listing the available hosts in a file, from which 10 successive processors were chosen. The master host at the end of 14-processor list was thus omitted. In the second test ("Static random") the master host was acting as the first stage in the pipeline, both reading images through GIMP, processing it and passing to the second stage. Especially for large images this becomes a bottleneck since the master host also saves the results to the disk. Finally, in the "Dynamic with shell" example, the remote shell was launched to start 10 slave node processes on least loaded nodes. It automatically omitted both the master host and the processor busy with text editing although seemingly idle. Compared to the "Static not loaded" case it is visible that even small additional loads in the static allocation scenario slow down the pipeline, justifying the use of the remote shell.

The best theoretical speed-up of the pipeline is strictly bounded and, assuming no overhead for communication which is more apparent for larger bitmaps, can be estimated as $\frac{N P}{N-1+P}(N=25, P=10)$ which approximates to $\frac{250}{34} \approx 7.35$. The time obtained for 1 processor and $25800 \times 600$ bitmaps and the 10-stage pipeline was 439s giving speed-up 5.49. This is due to costly communication and synchronization. It must be noted also that on 1 processor all the processes run concurrently which means that there are costly context switches and slow downs due to the GIMP's disk operations.

\section{Summary and Future Work}

We have presented software developments combining advanced pipelined filter processing of images selected in GIMP with parallel clusters showing improvement in the execution time when used with a load-aware remote shell rather than static process assignment. It is easy to select the proposed as well as add new filters to customize the graphic flow, for example, to perform the common sequence of operations on images transformed to thumbnails for WWW use, usually transformed from TIFF: adjust levels (can be pipelined itself), convert to the 16-bit format, adjust contrast, brightness, possibly saturation, scale the image, apply the unsharp mask, convert to JPEG.

As it is a more practical pipeline flow, we are planning to implement such a pipeline and execute it on a cluster of 128 2-processor machines at the TASK center in Gdansk, Poland as well. Note that the proposed approach can be used widely for pipelined image conversion for WWW gallery creation or assist in pipelined sequences of advanced graphic designers working with the GIMP. The implementation could also be extended to other popular applications like Adobe Photoshop.

\section{References}

1. Foster, I., Kesselman, C., Tuecke, S.: The Anatomy of the Grid: Enabling Scalable Virtual Organizations. International Journal of High Performance Computing Applications 15 (2001) 200-222 http://www.globus . org/research/papers/anatomy.pdf.

2. Globus: Fundamental Technologies Needed to Build Computational Grids (2003) http: //www.globus .org.

3. Sun Microsystems Inc.: Sun Grid Engine 5.3. Administration and User's Guide. (2002) http://wwws.sun. com/software/gridware/faq.html. 
4. Barak, A., La'adan, O.: The MOSIX Multicomputer Operating System for High Performance Cluster Computing. Journal of Future Generation Computer Systems 13 (1998) 361-372

5. Platform Computing Inc.: PLATFORM LSF, Intelligent, policy-driven batch application workload processing (2003) http: //www . platform. com/products/LSF/.

6. Bricker, A., Litzkow, M., Livny, M.: Condor Technical Summary. Technical report, Computer Sciences Department, University of Wisconsin-Madison (10/9/91)

7. Wilkinson, B., Allen, M.: Parallel Programming: Techniques and Applications Using Networked Workstations and Parallel Computers. Prentice Hall (1999)

8. Kepner, J.: Parallel Programming with MatlabMPI. MIT, Lexington, MA, U.S.A. (2003) http://www.1l.mit.edu/MatlabMPI/.

9. Marc Pawliger: Multithreading Photoshop (1997) http://www.reed.edu/ cosmo/ pt/tips/Multi.html.

10. Mainelli, T.: Two cpus in one? the latest pentium 4 chip reaches $3 \mathrm{ghz}$ and promises you a virtual second processor via intel's hyperthreading technology. PC World Magazine (Jan 2003)

11. Briggs, E.: Threaded Gimp Plugins (2003) http://nemo.physics.ncsu.edu/ briggs/gimp/.

12. Seinstra, F., Koelma, D., Geusebroek, J., Verster, F., Smeulders, A.: Efficient Applications in User Transparent Parallel Image Processing. In: Proceeding of International Parallel and Distributed Processing Symposium: IPDPS 2002 Workshop on Parallel and Distributed Computing in Image Processing, Video Processing, and Multimedia (PDIVM'2002), Fort Lauderdale, Florida, U.S.A. (2002) citeseer .nj .nec . com/552453.html.

13. Nicolescu, C., Jonker, P.: EASY-PIPE - An "EASY to Use" Parallel Image Processing Environment Based on Algorithmic Skeletons. In: Proceedings of the 15th International Parallel and Distributed Processing Symposium (IPDPS'01), Workshop on Parallel and Distributed Image Processing, Video Processing, and Multimedia (PDIVM'2001), San Francisco, California, USA (2001)

http://csdl . computer .org/comp/proceedings/ipdps/2001/0990/03/ 099030114aabs.htm.

14. Squyres, J.M., Lumsdaine, A., Stevenson, R.L.: A Toolkit for Parallel Image Processing. In: Proceedings of SPIE Annual Meeting Vol. 3452, Parallel and Distributed Methods for Image Processing II, San Diego (1998)

15. Oliveira, P., du Buf, H.: SPMD Image Processing on Beowulf Clusters: Directives and Libraries. In: Proceedings of International Parallel and Distributed Processing Symposium (IPDPS'03), Workshop on Parallel and Distributed Image Processing, Video Processing, and Multimedia (PDIVM'2003), Nice, France (2003)

http://csdl . computer.org/comp/proceedings/ipdps/2003/1926/00/ 19260230aabs.htm.

16. Czarnul, P.: Programming, Tuning and Automatic Parallelization of Irregular Divide-andConquer Applications in DAMPVM/DAC. International Journal of High Performance Computing Applications 17 (2003) 77-93

17. Czarnul, P.: Development and Tuning of Irregular Divide-and-Conquer Applications in DAMPVM/DAC. In: Recent Advances in Parallel Virtual Machine and Message Passing Interface. Number 2474 in Lecture Notes in Computer Science, Springer-Verlag (2002) 208216 9th European PVM/MPI Users' Group Meeting, Linz, Austria, September/October 2002, Proceedings.

18. Czarnul, P., Krawczyk, H.: Parallel Program Execution with Process Migration. In: International Conference on Parallel Computing in Electrical Engineering (PARELEC'00), Proceedings, Quebec, Canada (2000) 